\title{
VARIACIÓN DEL NÚMERO DE SEMILLAS EN FRUTOS DE 156 ESPECIES LEÑOSAS DEL ECUADOR
}

\author{
José Miguel Romero-Saritama ${ }^{\star \star}$, Magaly Germania Granda ${ }^{2}$ \\ ${ }^{1}$ Universidad Técnica Particular de Loja. Departamento de Ciencias Biológicas. \\ ${ }^{2}$ Universidad de las Fuerzas Armadas ESPE. Unidad de Seguridad Integrada. \\ * Autor para correspondencia: jmromero@utpl.edu.ec \\ Recibido: 2020/09/28 Aprobado: 2020/11/17 \\ DOl: https://doi.org/10.26621/XVI23.2020.12.A09.PUCESI.2550.6684
}

\section{RESUMEN}

El estudio de rasgos funcionales en semillas permite aumentar nuestro conocimiento sobre la morfología y biología reproductiva de los ambientes tropicales. Sin embargo, rasgos en semillas de plantas forestales han sido escasamente evaluados. El objetivo del presente trabajo fue determinar la variación del número de semillas por fruto en 156 especies leñosas distribuidas en diferentes hábitats del Ecuador. Para ello, se recolectó entre cinco y diez frutos de cinco individuos por especie. Se extrajo y cuantificó el número de semillas obtenidas en los frutos y se relacionó con el tipo de fruto, hábito y hábitat de las especies. Los resultados mostraron que las especies presentan desde una a más de 400 semillas por fruto. No obstante, la tendencia del $57 \%$ de las especies leñosas es producir menos de 10 semillas por fruto. Este rasgo mostró mayor rango de variación en la cantidad de semillas por fruto entre las especies de bosques húmedo y seco. Sin embargo, no se evidenció diferencias significativas con el tipo de fruto y hábito de la especie.

Palabras clave: bosques tropicales, conservación ex situ, especies leñosas, producción de semillas, recolección de semilla, variación interespecífica

\section{ABSTRACT}

The functional study seeds traits allows us to increase our knowledge about the morphology and reproductive biology in tropical environments. However, seeds traits in forest plants have been poorly evaluated. The objective of this work was to determine the variation in seeds number per fruit in 156 woody species distributed in different habitats of Ecuador. For that, between five and ten fruits were collected from five individuals per species. The seeds number obtained in the fruits was extracted and quantified and it was related to the fruit type, habit, and species. The results showed that the species present from one to more than 400 seeds per fruit. However, the tendency of $57 \%$ of woody species is to produce less than 10 seeds per fruit. This trait showed a greater range of variation in the amount of seeds per fruit between the wet and dry forest species. However, there is not significant differences they were evidenced with the fruit type and species habit.

Keywords: tropical forests, ex situ conservation, woody species, seed production, collection seeds, interspecific variation 


\section{INTRODUCCIÓN}

Los rasgos funcionales de las plantas son un reflejo de las estrategias ecológicas que permiten comprender el funcionamiento de las comunidades vegetales (Lohbeck et al., 2015). Dentro de estos rasgos, el tamaño, peso, formas y número de semillas han contribuido al estudio de los procesos de dispersión, colonización y establecimiento de las plántulas. Estos elementos constituyen, así, aspectos fundamentales dentro de los estudios ecológicos de cualquier hábitat (Dalling, 2002).

Conocer en las especies el número de semillas que producen los frutos puede ser un rasgo importante para determinar estrategias reproductivas de las plantas y para mejorar programas de recolección de semillas con fines de conservación ex situ. No obstante, este rasgo ha sido escasamente estudiado en ambientes tropicales. De los pocos trabajos realizados, se ha identificado que el número de semillas por fruto varía significativamente intra e Inter específicamente en especies de un remanente de bosque seco (Romero-Saritama y Pérez-Ruiz, 2016a). Asimismo, la variación de las semillas producidas en los frutos puede darse a nivel de variedades de una misma especie, como es el caso de Physalis peruviana (Peña et al., 2010). Por lo tanto, puede existir un alto grado de variación de semillas por frutos en especies dentro de un ecosistema (López-Palacios et al., 2015), en individuos de una misma especie, entre diferentes procedencias e incluso entre genotipos como en el caso de Jatropha curcas (Makkar et al., 2008). Adicionalmente, la cantidad de semillas producidas por especie tiene una implicación morfológica y reproductiva. Generalmente, especies con mayor cantidad de semillas presentan semillas más pequeñas y con menor peso (Baker et al., 1994), a diferencia de aquellas que producen pocas semillas pero de mayor tamaño y mejor capacidad para germinar (Jakobsson y Eriksson, 2000; Lavorel y Garnier, 2002).

Por otro lado, identificar la variación de la cantidad de semillas que se producen dentro de los frutos en las especies leñosas provee información importante a la hora de proponer programas y estrategias de conservación ex situ de germoplasma vegetal, especialmente en los procesos de recolección en campo (Romero-Saritama y Pérez-Ruiz, 2016b), ya que el esfuerzo y tiempo dedicados a la recolectar frutos (sea con muchas o sea con pocas semillas), para su conservación, pueden ser diferente para cada especie. Sin embargo, esta información ha sido escasamente explorada a nivel de especies tropicales o se encuentra en informes técnicos no publicados. Con el fin de llenar vacíos de información morfológica y biológica de las semillas de especies vegetales del trópico, el objetivo del presente trabajo fue analizar la variación del número de semillas por fruto de especies leñosas distribuidas en seis ecosistemas del Ecuador, permitiendo así tener más elementos para la toma de decisiones al momento de la recolección de semillas con fines de conservación ex situ.

\section{MATERIALES Y MÉTODOS}

Se trabajó con 156 especie leñosas entre árboles y arbustos, pertenecientes a 47 familias distribuidas en seis ecosistemas del Ecuador. El taxón Fabaceae fue el más representativo de la muestra, con 53 especies. Durante el período del 2014 al 2017 se recolectaron de cinco a diez frutos maduros de cinco individuos por cada especie. Los frutos fueron tomados directamente de la planta durante su fase de dispersión natural, evidenciando que los mismos se encuentren en buen estado fitosanitario, no presenten daño mecánico o posean alguna abertura que pudo implicar la salida de semillas. Durante la recolección, se determinó el hábito (árbol o arbusto) de las plantas y se identificó su hábitat gene- ral (bosque seco, bosque húmedo, bosque montano, matorral húmedo, matorral seco o páramo). Los frutos fueron analizados en el laboratorio de semillas de la Universidad Técnica Particular de Loja, donde primeramente se determinó el tipo de fruto según su consistencia (seco 0 carnoso). Posteriormente, se extrajo y contabilizó el número de semillas dentro de los frutos y se obtuvo un promedio para cada especie. Según el promedio de semillas por fruto, se clasificó a las especies en siete categorías: a) especies con 1 semilla, b) de 2 a 5, c) de 6 a 10, d) de 11 a 20 , e) de 21 a 50, f) de 51 a 100 y g) especies con más de 100 semillas. Adicionalmente, en el estudio, se incluyó la información del número de semillas por fruto de especies leñosas de los bosques secos del sur del Ecuador estudiadas por Romero-Saritama y Pérez- Ruiz (2016a).

Para determinar diferencias significativas del número de semillas por fruto entre el hábito y tipo de fruto, se realizó la prueba de T de Student; La prueba "t" de Student es un tipo de estadística deductiva. Se utiliza para determinar si hay una diferencia significativa entre las medias de dos grupos. Con toda la estadística deductiva, asumimos que las variables dependientes tienen una distribución normal. En este sentido, utilizamos ANOVA (análisis de la variación unidireccional) de un factor para comparar el número de semillas con el hábitat de la especie. Las comparaciones post-hoc se realizaron mediante pruebas de Tukey con un nivel de confianza de 95\%.

\section{RESULTADOS Y DISCUSIÓN}

Se determinó un alto rango de variación en el número de semillas por fruto entre las especies estudiadas (figura 1). De este modo, se encontraron especies que producen frutos desde una semilla, como por ejemplo en; Cordia lutea (Boraginaceae), Celtis iguanaea (Cannabaceae), Schizolobium parahyba (Fabaceae), Juglans neotropica (Juglandaceae), hasta especies que producen más de 400 semillas, como en el caso de Ochroma pyramidale (tabla 1). No obstante, el $81 \%$ de las especies tiende a producir menos de 50 semillas en los frutos (figura 1). La variación encontrada en el número de semillas por fruto puede deberse, por un lado, a la competencia que se da entre embriones cuando los recursos maternos son limitados durante su desarrollo (Wolf et al., 1986), o por una respuesta adaptativa de las especies ante la carencia o cambios en los recursos disponibles en la planta (Vaughton y Ramsey, 1998; Ayala-Cordero et al., 2004). Además, la variación también puede ser originada por presiones ambientales en el hábitat donde se desarrollan cada una de las especies (Vaughton y Ramsey, 1998).

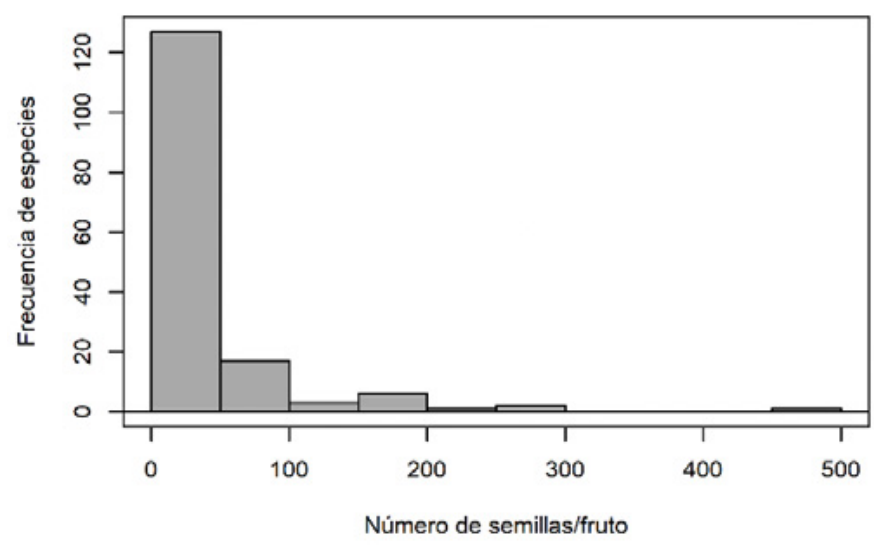

Figura 1. Variación y frecuencia de especies según el número de semillas por fruto. 
Tabla 1. Listado de especies en estudio, características y número de semillas por fruto.

\begin{tabular}{|c|c|c|c|c|}
\hline Familia & Especie & Hábito & Hábitat & $\begin{array}{l}\text { Número de } \\
\text { semillas }\end{array}$ \\
\hline Amaranthaceae & Alternanthera lanceolata (Benth.) Schinz & Arbusto & BS & $8 \pm 0.9$ \\
\hline Anacardiaceae & Spondia purpurea L. & Arbusto & BS & $1 \pm 0$ \\
\hline Anacardiaceae & Schinus molle L. & Árbol & BM & $1 \pm 0$ \\
\hline Anacardiaceae & Mauria suaveolens Poepp. \& Endl. & Árbol & BS & $18 \pm$ \\
\hline Anacardiaceae & Loxopterygium huasango Spruce ex Engl. & Árbol & BS & $1 \pm 0$ \\
\hline Annonaceae & Annona muricata L. & Árbol & BS & $55 \pm 16$ \\
\hline Araliaceae & Oreopanax ecuadorensis Seem. & Árbol & BM & $4 \pm 0.8$ \\
\hline Arecaceae & Bactris gasipaes Kunth & Árbol & $\mathrm{BH}$ & $1 \pm 0$ \\
\hline Arecaceae & Iriartea deltoidea Ruiz \& Pav. & Árbol & $\mathrm{BH}$ & $1 \pm 0$ \\
\hline Arecaceae & Mauritia flexuosa L.f. & Árbol & $\mathrm{BH}$ & $1 \pm 0$ \\
\hline Arecaceae & Socratea exorrhiza (Mart.) H. Wendl. & Árbol & $\mathrm{BH}$ & $1 \pm 0$ \\
\hline Asteraceae & Ambrosia arborescens Mill. & Arbusto & $\mathrm{MH}$ & $1 \pm 0$ \\
\hline Asteraceae & Taraxacum officinale L. & Arbusto & BM & $77 \pm 4.1$ \\
\hline Berberidaceae & Berberis hallii Hieron. & Arbusto & BM & $4 \pm 0$ \\
\hline Betulaceae & Alnus acuminata Kunth & Árbol & BM & $156 \pm 10$ \\
\hline Bignoniaceae & Delostoma roseum (H. Karst. \& Triana) K. Schum. & Arbusto & BS & $54 \pm 3.0$ \\
\hline Bignoniaceae & Jacaranda copaia (Aubl.) D. Don & Árbol & $\mathrm{BH}$ & $83 \pm 8.0$ \\
\hline Bignoniaceae & Jacaranda mimosifolia D. Don & Árbol & BS & $69 \pm 10$ \\
\hline Bignoniaceae & Tecoma stans (L.) Juss. ex Kunth & Arbusto & BM & $81 \pm 2.0$ \\
\hline Bignoniaceae & Tecoma castaneifolia (D. Don) Melch. & Árbol & BM & $37 \pm 4.0$ \\
\hline Bixaceae & Bixa orellana $\mathrm{L}$. & Árbol & $\mathrm{BH}$ & $46 \pm 12$ \\
\hline Bixaceae & Cochlospermum vitifolium (Willd.) Spreng. & Árbol & BS & $105 \pm 10$ \\
\hline Boraginaceae & Cordia macrantha Chodat & Árbol & BS & $1 \pm 0$ \\
\hline Boraginaceae & Cordia lutea Lam. & Arbusto & BS & $1 \pm 0$ \\
\hline Boraginaceae & Cordia macrocephala (Desv.) Kunth & Arbusto & BS & $1 \pm 0$ \\
\hline Boraginaceae & Cordia alliodora (Ruiz \& Pav.) Oken & Árbol & BS & $1 \pm 0$ \\
\hline Buddlejaceae & Buddleja bullata Kunth & Árbol & BS & $52 \pm 3.0$ \\
\hline Burseraceae & Bursera graveolens (Kunth) Triana \& Planch. & Árbol & BS & $1 \pm 0$ \\
\hline Cannabaceae & Celtis iguanaea (Jacq.) Sarg. & Arbusto & BS & $1 \pm 0$ \\
\hline Cannabaceae & Trema micrantha (L.) Blume & Árbol & BS & $1 \pm 0$ \\
\hline Caprifoliaceae & Sambucus peruviana Kunth & Árbol & BM & $4 \pm 0.7$ \\
\hline Caprifoliaceae & Sambucus nigra L. & Árbol & BS & $4 \pm 0.6$ \\
\hline Caricaceae & Vasconcellea pubescens A. DC. & Árbol & BM & $65 \pm 10$ \\
\hline Cecropiaceae & Pourouma cecropiifolia Mart. & Árbol & $\mathrm{BH}$ & $1 \pm 0$ \\
\hline Cecropiaceae & Cecropia obtusifolia Bertol. & Árbol & $\mathrm{BH}$ & $1 \pm 0$ \\
\hline Combretaceae & Terminalia amazonia (J.F. Gmel.) Exell & Árbol & $\mathrm{BH}$ & $1 \pm 0$ \\
\hline Combretaceae & Bucida buceras L. & Arbusto & BS & $16 \pm$ \\
\hline Combretaceae & Terminalia oblonga (Ruiz \& Pav.) Steud. & Árbol & $\mathrm{BH}$ & $1 \pm 0$ \\
\hline Combretaceae & Terminalia valverdeae A.H. Gentry & Árbol & BS & $1 \pm 0$ \\
\hline Convolvulaceae & Ipomoea carnea Jacq. & Arbusto & BS & $4 \pm 0$ \\
\hline Convolvulaceae & Ipomoea pauciflora M. Martens \& Galeotti & Arbusto & BS & $4 \pm 0$ \\
\hline Elaeocarpaceae & Vallea stipularis $\mathrm{L}$. f. & Árbol & BS & $2 \pm 0$ \\
\hline
\end{tabular}




\begin{tabular}{|c|c|c|c|c|}
\hline Ericaceae & Pernettya prostrata (Cav.) DC. & Arbusto & PA & $186 \pm 25^{*}$ \\
\hline Ericaceae & Vaccinium floribundum Kunth & Arbusto & $\mathrm{BH}$ & $132 \pm 29$ \\
\hline Erythroxylacea & Erythroxy/um glaucum 0.E. Schulz & Árbol & BS & $1 \pm 0$ \\
\hline Euphorbiaceae & Ricinus communis $\mathrm{L}$. & Arbusto & BS & $3 \pm 0$ \\
\hline Euphorbiaceae & Caryodendron orinocense $\mathrm{H}$. Karst. & Árbol & $\mathrm{BH}$ & $1 \pm 0$ \\
\hline Euphorbiaceae & Croton wagneri Müll. Arg. & Arbusto & MS & $3 \pm 0$ \\
\hline Euphorbiaceae & Croton elegans Kunth & Arbusto & BS & $3 \pm 0$ \\
\hline Euphorbiaceae & Hieronyma macrocarpa Müll. Arg. & Árbol & BM & $1 \pm 0$ \\
\hline Euphorbiaceae & Hura crepitans $\mathrm{L}$. & Árbol & BS & $16 \pm 0.8$ \\
\hline Fabaceae & Acacia dealbata Link & Arbusto & BM & $6 \pm 0.5$ \\
\hline Fabaceae & Acacia macracantha Humb. \& Bonpl. ex Willd. & Árbol & BS & $12 \pm 3$ \\
\hline Fabaceae & Acacia melanoxylon R. Br. & Árbol & BS & $6 \pm 1.0$ \\
\hline Fabaceae & Acacia horrida Span. & Árbol & BS & $12 \pm 1.6$ \\
\hline Fabaceae & Acacia sp. & Árbol & BM & $6 \pm 0.5$ \\
\hline Fabaceae & Albizia multiflora (Kunth) Barneby \& J.W. Grimes & Árbol & BS & $6 \pm 1.0$ \\
\hline Fabaceae & Albizia guachapele (Kunth) Dugand & Árbol & BS & $21 \pm 5.0$ \\
\hline Fabaceae & Cassia canescens Kunth & Árbol & BM & $29 \pm 3.1$ \\
\hline Fabaceae & Cassia tomentosa L. f. & Arbusto & BM & $22 \pm 1.3$ \\
\hline Fabaceae & Caesalpinia glabrata Kunth & Árbol & BS & $7 \pm 0.9$ \\
\hline Fabaceae & Caesalpinia spinosa (Molina) Kuntze & Árbol & BS & $10 \pm 0.6$ \\
\hline Fabaceae & Calliandra angustifolia Spruce ex Benth. & Arbusto & $\mathrm{BH}$ & $5 \pm 0.5$ \\
\hline Fabaceae & Cajanus cajan (L.) Huth & Arbusto & BM & $7 \pm 1.4$ \\
\hline Fabaceae & Centrolobium ochroxylum Rose ex Rudd & Árbol & BS & $2 \pm 0.5$ \\
\hline Fabaceae & Crotalaria micans Link & Arbusto & $\mathrm{BH}$ & $23 \pm 1.8$ \\
\hline Fabaceae & Clitoria brachystegia Benth. & Árbol & BS & $11 \pm 2.3$ \\
\hline Fabaceae & Chloroleucon mangense (Jacq.) Britton \& Rose & Árbol & BS & $9 \pm 10$ \\
\hline Fabaceae & Delonix regia (Bojer ex Hook.) Raf. & Árbol & BS & $26 \pm 4.0$ \\
\hline Fabaceae & Erythrina amazónica Krukoff & Árbol & $\mathrm{BH}$ & $10 \pm 2.0$ \\
\hline Fabaceae & Erythrina coralloides DC. & Árbol & $\mathrm{BH}$ & $13 \pm 1.3$ \\
\hline Fabaceae & Erythrina smithiana Krukoff & Árbol & BS & $14 \pm 1.2$ \\
\hline Fabaceae & Erythrina uleiHarms & Árbol & $\mathrm{BH}$ & $5 \pm 0.9$ \\
\hline Fabaceae & Erythrina poeppigiana (Walp.) 0.F. Cook & Árbol & $\mathrm{BH}$ & $7 \pm 0.8$ \\
\hline Fabaceae & Genista monspessulana (L.) L.A.S. Johnson & Arbusto & BM & $4 \pm 0.7$ \\
\hline Fabaceae & Geoffroea spinosa Jacq. & Árbol & BS & $1 \pm 0$ \\
\hline Fabaceae & Haematoxylum campechianum $\mathrm{L}$. & Árbol & BS & $6 \pm 0.7$ \\
\hline Fabaceae & Inga edulis Mart. & Árbol & BM & $14 \pm 4.0$ \\
\hline Fabaceae & Inga insignis Kunth & Árbol & $\mathrm{MH}$ & $7 \pm 1.3$ \\
\hline Fabaceae & Leucaena leucocephala (Lam.) de Wit & Árbol & BS & $19 \pm 3.7$ \\
\hline Fabaceae & Leucaena trichodes (Jacq.) Benth. & Arbusto & BS & $10 \pm 1.3$ \\
\hline Fabaceae & Lonchocarpus utilis A.C. Sm. & Arbusto & $\mathrm{BH}$ & $68 \pm 12$ \\
\hline Fabaceae & Machaerium capote Triana ex Dugand & Árbol & BS & $1 \pm 0$ \\
\hline Fabaceae & Machaerium millei Standl. & Árbol & BS & $1 \pm 0$ \\
\hline Fabaceae & Mimosa quitensis Benth. & Arbusto & BM & $6 \pm 1.9$ \\
\hline Fabaceae & Mimosa pudica L. & Arbusto & $\mathrm{BH}$ & $4 \pm 0.6$ \\
\hline Fabaceae & Myroxylon peruiferum L. f. & Árbol & BS & $1 \pm 0$ \\
\hline Fabaceae & Ormosia coccinea (Aubl.) Jacks. & Árbol & $\mathrm{BH}$ & $3 \pm 1.3$ \\
\hline Fabaceae & Piscidia carthagenensis Jacq. & Árbol & BS & $5 \pm 0.7$ \\
\hline Fabaceae & Platymiscium pinnatum (Jacq.) Dugand & Árbol & BH & $1 \pm 0$ \\
\hline
\end{tabular}




\begin{tabular}{|c|c|c|c|c|}
\hline Fabaceae & Platymiscium sp. & Árbol & BS & $1 \pm 0$ \\
\hline Fabaceae & Prosopis juliflora (Sw.) DC. & Árbol & BS & $18 \pm 1.7$ \\
\hline Fabaceae & Samanea saman (Jacq.) Merr. & Árbol & BS & $22 \pm 3.3$ \\
\hline Fabaceae & $\begin{array}{l}\text { Senna mollissima (Humb. \& Bonpl. ex Willd.) H.S. Irwin \& } \\
\text { Barneby }\end{array}$ & Árbol & BS & $26 \pm 5.0$ \\
\hline Fabaceae & Senna spectabilis (DC.) H.S. Irwin \& Barneby & Árbol & BS & $81 \pm 3.2$ \\
\hline Fabaceae & Senna multiglandulosa (Jacq.) H.S. Irwin \& Barneby & Arbusto & $\mathrm{BH}$ & $27 \pm 9.8$ \\
\hline Fabaceae & Senna didymobotrya (Fresen.) H.S. Irwin \& Barneby & Arbusto & BM & $9 \pm 1.7$ \\
\hline Fabaceae & Spartium junceum L. & Arbusto & BS & $13 \pm 1.8$ \\
\hline Fabaceae & Schizolobium parahyba (Vell.) S.F. Blake & Árbol & BS & $1 \pm 0$ \\
\hline Fabaceae & Spartium sp. & Arbusto & $\mathrm{MH}$ & $8 \pm 1.0$ \\
\hline Fabaceae & Tamarindus indica L. & Árbol & BS & $5 \pm 1.8$ \\
\hline Fabaceae & Tipuana tipu (Benth.) Kuntze & Árbol & BS & $37 \pm 5.5$ \\
\hline Juglandaceae & Juglans regia L. & Árbol & BM & $1 \pm 0$ \\
\hline Juglandaceae & Juglans neotropica Diels & Árbol & $\mathrm{BH}$ & $1 \pm 0$ \\
\hline Lamiaceae & Tectona grandis L. f. & Árbol & BS & $1 \pm 0$ \\
\hline Lamiaceae & Vitex gigantea Kunth & Árbol & $\mathrm{BH}$ & $1 \pm 0$ \\
\hline Lamiaceae & Aegiphila ferruginea Hayek \& Spruce & Árbol & BM & $14 \pm 2.1$ \\
\hline Loganiaceae & Potalia amara Aubl. & Arbusto & $\mathrm{BH}$ & $20 \pm 2.0$ \\
\hline Malvaceae & Cavanillesia platanifolia (Bonpl.) Kunth & Árbol & BS & $1 \pm 0$ \\
\hline Malvaceae & Ceiba trischistandra (A. Gray) Bakh. & Árbol & BS & $84 \pm 10$ \\
\hline Malvaceae & Gossypium barbadense L. & Arbusto & $\mathrm{BH}$ & $40 \pm 20$ \\
\hline Malvaceae & Ochroma pyramidale (Cav. ex Lam.) Urb. & Árbol & $\mathrm{BH}$ & $476 \pm 90$ \\
\hline Malvaceae & Spirotheca rimbachii Cuatrec. & Árbol & $\mathrm{BH}$ & $38 \pm 4.5$ \\
\hline Melastomataceae & Brachyotum ledifolium (Desr.) Triana & Arbusto & BM & $133 \pm 18$ \\
\hline Melastomataceae & Tibouchina ochypetala (Ruiz \& Pav.) Baill. & Arbusto & $\mathrm{BH}$ & $38 \pm 12$ \\
\hline Melastomataceae & Miconia papillosa (Desr.) Naudin & Arbusto & $\mathrm{BH}$ & $193 \pm 20$ \\
\hline Meliaceae & Cedrela odorata $\mathrm{L}$. & Árbol & $\mathrm{BH}$ & $34 \pm 4.2$ \\
\hline Meliaceae & Guarea macrophylla Vahl & Árbol & BS & $3 \pm 0.7$ \\
\hline Meliaceae & Guarea guidonia (L.) Sleumer & Árbol & $\mathrm{BH}$ & $3 \pm 0.7$ \\
\hline Meliaceae & Trichilia hirta L. & Arbusto & BS & $3 \pm 0$ \\
\hline Moraceae & Castilla elastica Sessé ex Cerv. & Árbol & BM & $17 \pm 1.3$ \\
\hline Moraceae & Clarisia racemosa Ruiz \& Pav. & Árbol & $\mathrm{BH}$ & $1 \pm 0$ \\
\hline Moraceae & Maclura tinctoria (L.) D. Don ex Steud. & Árbol & $\mathrm{BH}$ & $61 \pm 31$ \\
\hline Myrsinaneae & Myrsine andina (Mez) Pipoly & Arbusto & BM & $1 \pm 0$ \\
\hline Myrtaceae & Myrcianthes leucoxyla (Ortega) McVaugh & Árbol & BM & $3 \pm 0.9$ \\
\hline Myrtaceae & Myrcianthes hallii (0. Berg) McVaugh & Árbol & BM & $1 \pm 0$ \\
\hline Myrtaceae & Psidium guajava $\mathrm{L}$. & Árbol & $\mathrm{BH}$ & $179 \pm 10$ \\
\hline Myrtaceae & Luma apiculata (DC.) Burret & Arbusto & BS & $2 \pm 0.2$ \\
\hline Myrtaceae & Eugenia stipitata McVaugh & Arbusto & $\mathrm{BH}$ & $7 \pm 1.9$ \\
\hline Myrtaceae & Myrcianthes hallii (0. Berg) McVaugh & Árbol & BM & $2 \pm 0.7$ \\
\hline Myrtaceae & Myrcianthes rhopaloides (Kunth) McVaugh & Árbol & $\mathrm{BH}$ & $1 \pm 1$ \\
\hline Myrtaceae & Psidium guineense $\mathrm{Sw}$. & Árbol & BS & $48 \pm 15$ \\
\hline Nyctaginaceae & Pisonia aculeata $\mathrm{L}$. & Árbol & BS & $1 \pm 0$ \\
\hline Oleaceae & Chionanthus pubescens Kunth & Árbol & BM & $1 \pm 0$ \\
\hline Phytolaccaceae & Phytolacca sp. & Arbusto & $\mathrm{BH}$ & $15 \pm 2.0$ \\
\hline Polygonaceae & Triplaris cumingiana Fisch. \& C.A. Mey. & Árbol & BS & $1 \pm 0$ \\
\hline Polygalaceae & Monnina crassifolia (Bonpl.) Kunth & Arbusto & BM & $1 \pm 0$ \\
\hline
\end{tabular}




\begin{tabular}{llllc} 
Polygonaceae & Coccoloba ruiziana Lindau & Árbol & BS & $1 \pm 0$ \\
\hline Proteaceae & Oreocallis grandiflora (Lam.) R. Br. & Árbol & BS & $12 \pm 0.1$ \\
\hline Pytolacaceae & Gallesia integrifolia (Spreng.) Harms & Árbol & BS & $1 \pm 0$ \\
Rhamnaceae & Ziziphus thyrsiflora Benth. & Árbol & BS & $1 \pm 0$ \\
\hline Rosaceae & Rubus glaucus Benth. & Arbusto & BM & $71 \pm 6$ \\
Rosaceae & Prunus serotina Ehrh. & Árbol & BH & $1 \pm 0$ \\
Rubiaceae & Genipa americana L. & Árbol & BS & $55 \pm 6.3$ \\
\hline Rubiaceae & Condaminea corymbosa (Ruiz \& Pav.) DC. & Árbol & BH & $130 \pm 17$ \\
\hline Rubiaceae & Morinda citrifolia L. & Árbol & BH & $39 \pm 4.2$ \\
\hline Rubiaceae & Borojoa sorbilis (Ducke) Cuatrec. & Árbol & BM & $300 \pm 40$ \\
\hline Sapindaceae & Sapindus saponaria L. & Árbol & BS & $1 \pm 0$ \\
Sapindaceae & Dodonaea viscosa Jacq. & Arbusto & BS & $3 \pm 0.4$ \\
Solanaceae & Brugmansia arbórea (L.) Lagerh. & Arbusto & BM & $45 \pm 4$ \\
Solanaceae & Brugmansia sanguinea (Ruiz \& Pav.) D. Don & Arbusto & BM & $218 \pm 22$ \\
\hline Solanaceae & Brugmansia suaveolens (Humb. \& Bonpl. ex Willd.) Sweet & Arbusto & BM & $124 \pm 7.2$ \\
Solanaceae & Datura stramonium L. & Arbusto & BH & $276 \pm 25$ \\
\hline Solanaceae & Physalis peruviana L. & Arbusto & BM & $58 \pm 9.1$ \\
Sterculiaceae & Guazuma ulmifolia Lam. & Árbol & BS & $79 \pm 12$ \\
\hline Tiliaceae & Apeiba membranacea Spruce ex Benth. & Árbol & BH & $159 \pm 25$ \\
\hline
\end{tabular}

Nota: $\mathrm{BS}=$ Bosque seco, $\mathrm{BM}=\mathrm{B}$ osque montano, $\mathrm{BH}=\mathrm{B}$ osque húmedo, $\mathrm{MH}=$ Matorral húmedo, MS=Matorral seco,

$\mathrm{P}=$ Páramo * Según Romero-Saritama y Cueva-0jeda (2020).

Al determinar la proporción de especies de acuerdo con los rangos establecidos por el número de semillas por fruto, la mayoría de las especies presenta una semilla por fruto, mientras que solamente el $8 \%$ de especies producen frutos que pueden contener más de 100 semillas (figura 2A), como el caso de A. acuminata (Betulaceae) y 0. pyramidale (Malvaceae) que producen un promedio de $156 \pm 10$ y $476 \pm 90$ semillas por fruto respectivamente. Las variaciones del número de semillas proporcionan un ejemplo clásico de una compensación evolutiva resultante del esfuerzo y asignación reproductiva, en la que la selección favorece a unas pocas semillas grandes o muchas semillas pequeñas (Venable, 1992; Jakobsson y Eriksson, 1999; Ramirez, 1993), hecho que posteriormente se refleja en un mayor o mejor potencial de germinación.

Adicionalmente, el número de semillas por fruto y por especie puede estar relacionado con la capacidad de sobrevivencia de las semillas y plántulas in situ. En plantas que producen gran número de semillas pequeñas (por ejemplo, Psidium guajava) y cuyas semillas se diseminan de una forma más amplia, éstas tienen pocas posibilidades de sobrevivir y germinar debido a la escasez de recursos nutricionales disponibles dentro de las mismas (Kshitij, 2012). En cambio, en plantas que producen menor número de semillas, pero de mayor peso, aunque la distancia de diseminación puede ser más corta, estas producen plántulas más resistentes con una reserva de nutrientes que le permitirá sobrevivir a las adversidades ambientales y ecológicas de los ecosistemas (Kshitij, 2012).

Por otra parte, determinar el número de semillas/fruto en cada especie podría tener grandes implicaciones al momento de planificar jornadas de recolección de semillas con fines de conservación ex situ. Cabe reseñar que, en Ecuador, la escasa información e investigación en semillas forestales ha creado una gran brecha de conocimiento para la recolección (Romero-Saritama, 2018). Identificar la cantidad de semillas en los frutos podría ayudar a optimizar recursos económicos, personal y esfuerzos en la recolección de semillas. Por ejemplo, si deseamos conservar un promedio de 10 mil semillas por especie, resulta obvio que, para aquellas especies que producen una semilla por fruto, el esfuerzo de recolecciónes mayor, porque se deberá recolectar justamente 10 mil frutos.
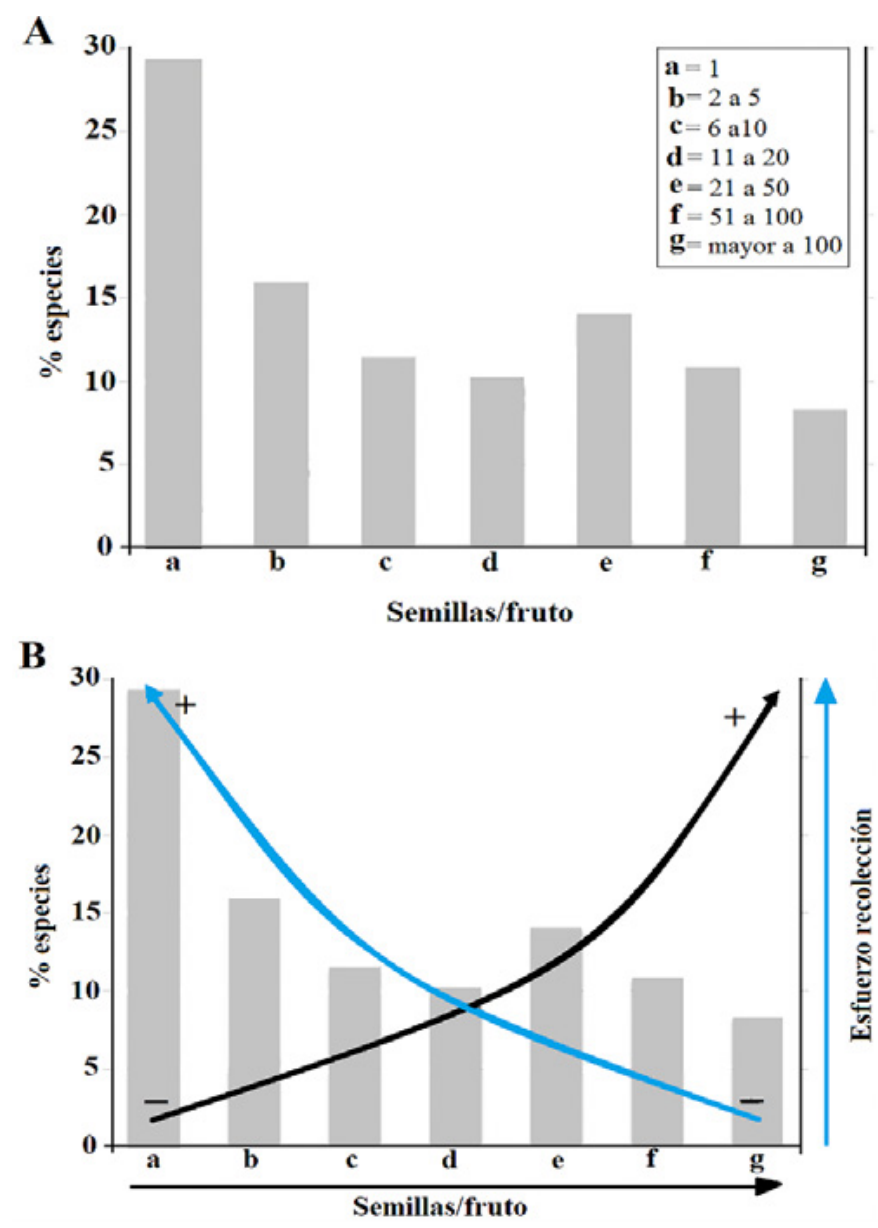

Figura 2. A. Proporción de especies según número de semillas por fruto en base a las 7 categorías. B. Proporción inversa entre el número de semillas/fruto y esfuerzo de recolección de semillas. 
En cambio, si existe una especie que produce 10 semillas/fruto, se deberá colectar 1.000 frutos. Y así, mientas mayor cantidad de semillas en los frutos, el tiempo y esfuerzo de recolección disminuye (figura 2B). Ello tiene como consecuencia la mejora y optimización de los procesos de recolección de semillas para la conservación ex situ.

Al analizar el número de semillas por fruto en base al hábito de la especie, no se encontró diferencias significativas entre los árboles y arbustos. Sin embargo, en el caso de los árboles, la tendencia de la mayoría de las especies es presentar menos de 50 semillas por fruto (figura 3) a excepción de Ochroma pyramidale que puede llegar a tener 476 semillas. En cambio, los arbustos tienden a un máximo de 276 semillas, aunque, igualmente, la mayoría de las especies producen menos de 50 semillas por fruto. Estos resultados concuerdan con Hernández-Verdugo et al., (2008) y Montejo Valdés et al., (2015) quienes sostienen que las variaciones en el número de semillas por fruto no guardan relación con el hábito de las especies, sino con la cantidad de nutrientes, el hábitat y al esfuerzo que realice la planta para reproducirse.

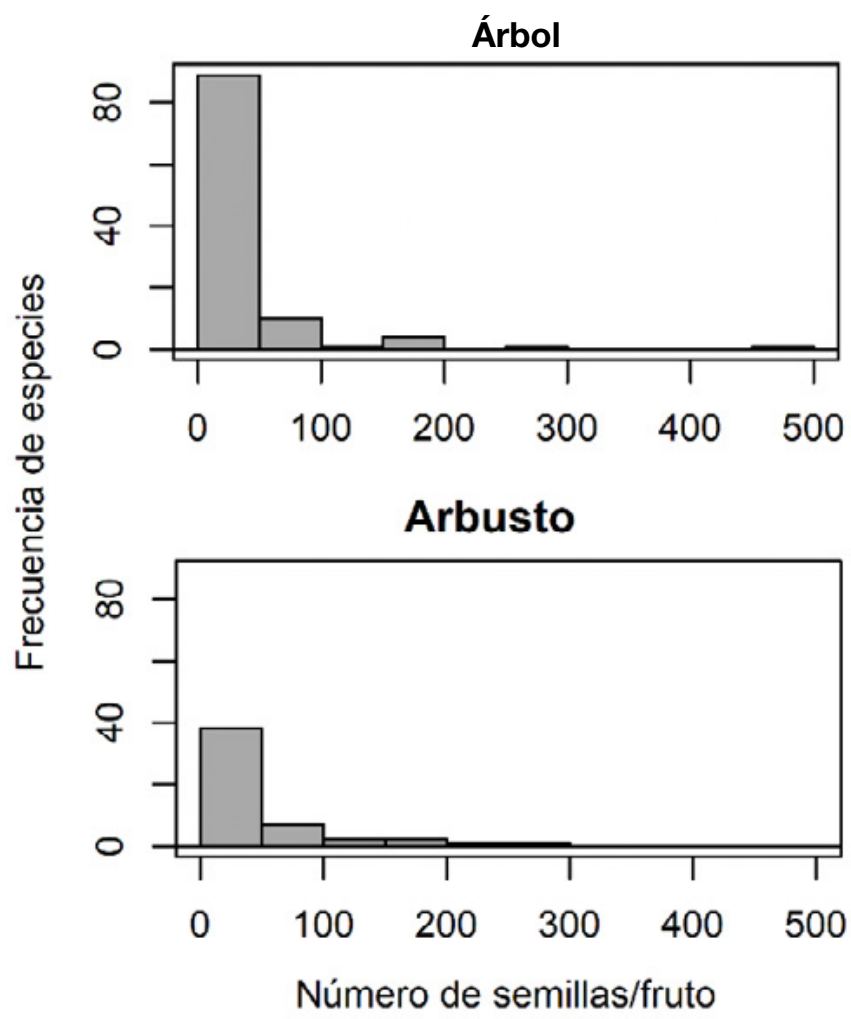

Figura 3. Variación del número de semillas por fruto en las especies según su hábito.

Al realizar los análisis del número de semillas por frutos con base en el hábitat de la especie, se evidenció diferencias significativas solamente entre bosque húmedo y bosque seco $(p=0.03)$. En el primero, las especies producen entre 1 a 476 semillas por fruto, a diferencia del bosque seco donde se encontró que las especies contienen entre 1 a 150 semillas por fruto (Figura 4). Para los ecosistemas de páramo, matorral seco y húmedo no se encontró diferencias en la producción de semillas por fruto. Posiblemente, por la poca cantidad de especies incluidas en el estudio (ver tabla 1).
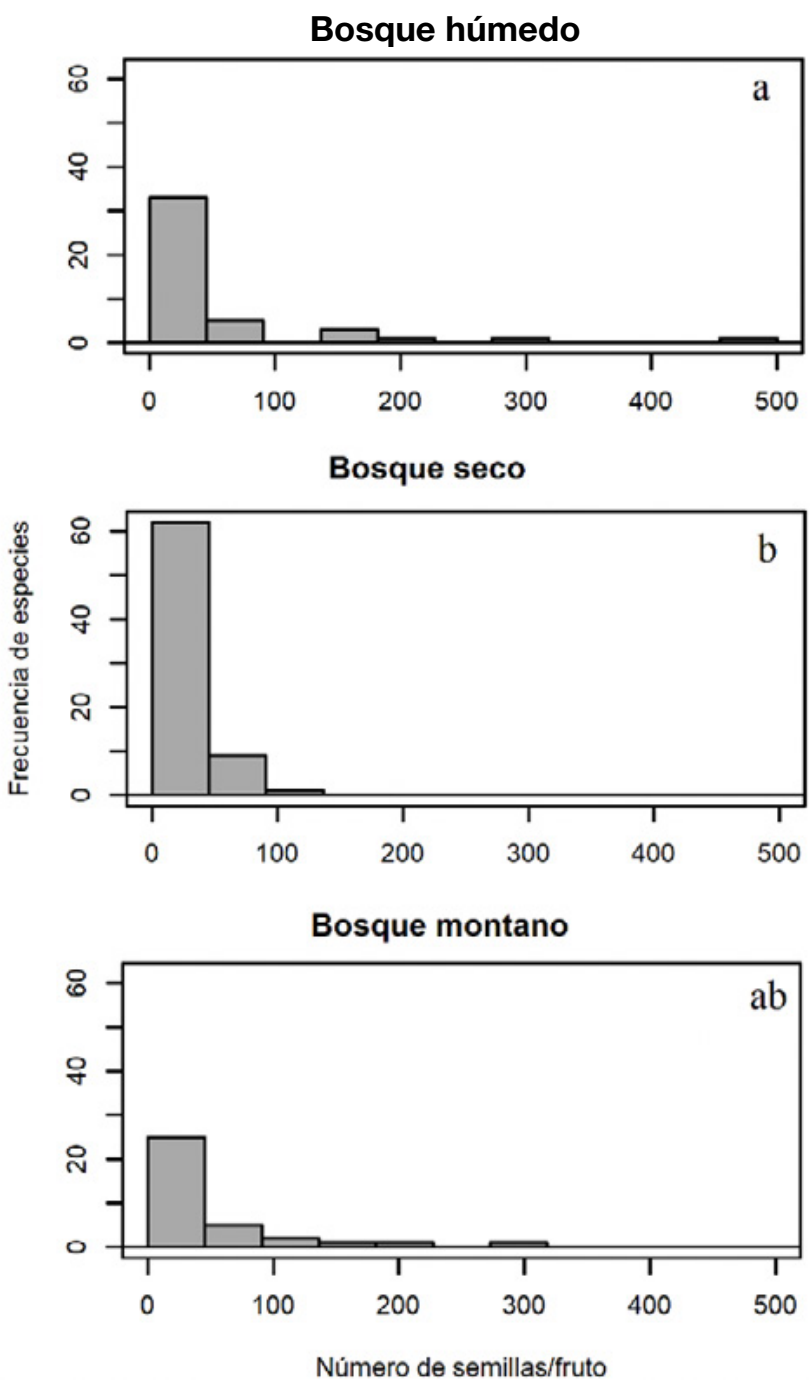

Figura 4. Frecuencia de especies de acuerdo con el número de semillas por fruto según el ecosistema. En la figura se muestra aquellos que, por la cantidad de especies fue posible el análisis. Las letras diferentes dentro de la figura muestran las diferencias significativas entre los ecosistemas (Tukey, 0.05).

Como se evidencia en la figura 4, las especies de bosque seco contienen una menor cantidad de semillas por fruto que especies adaptadas a condiciones ambientales de mayor humedad (bosque montano y húmedo). Una menor cantidad de semillas puede deberse a que la estacionalidad ambiental afecta los patrones de producción de semillas (Gritti et al., 2010). En ambientes donde existe escasez de lluvia, las especies posiblemente optarían por reducir el número de semillas por fruto a fin de optimizar recursos y aprovecharlos en la germinación durante el corto periodo de humedad (Romero-Saritama y Pérez-Ruiz, 2016a). Además, en condiciones ambientales desfavorables, las especies tienden a sufrir "abortos de semillas" durante su desarrollo en los frutos inmaduros, provocando una disminución en el número de semillas/fruto (Brancalion y Rodriguez, 2014). En cambio, en hábitats donde hay una gran competencia por los recursos ambientales como en el caso de los bosques húmedos y montanos-, la abundancia de semillas puede favorecer la dispersión de las especies. Al producir más semillas, puede existir una alta probabilidad posibilidad de ser mayormente interceptadas por algún agente dispersor y ser llevadas así a lugares de mejores condiciones para establecerse. No obstante, es necesario tomar en cuenta muchos factores involucrados en la ecología de la dispersión de semillas (Gentry, 1982). 
Ante la posibilidad de encontrar un mayor número de semillas por fruto en las especies forestales presentes en los bosques húmedos, es importante aclarar que ello, por una parte, facilita la recolección de una gran cantidad de semillas para su conservación. No obstante, esta compensación se podría ver retrasada por las condiciones de humedad ambiental, altura y estructura de la vegetación. Existe pues una fuerte diferencia respecto a las especies de bosques secos, donde, a pesar de producirse menos semillas por frutos, existe mejores condiciones para la recolección de semillas, especialmente en la temporada seca.

Al analizar el número de semillas por fruto de acuerdo con el tipo de fruto, se determinó que las especies con frutos secos presentaron mayor número, a diferencia de los frutos carnosos que contuvieron como máximo 300 semillas por fruto (figuras 5). Sin embargo, los análisis estadísticos mostraron que no existen diferencias significativas entre el tipo de fruto con relación al número de semillas/fruto. Estos resultados refuerzan lo afirmado anteriormente, esto es, que el número de semillas por fruto está en dependencia por la disponibilidad de recursos con que cuenta la planta para reproducirse y a factores genéticos que son particulares de cada especie (Sharma y Kumar, 2013).

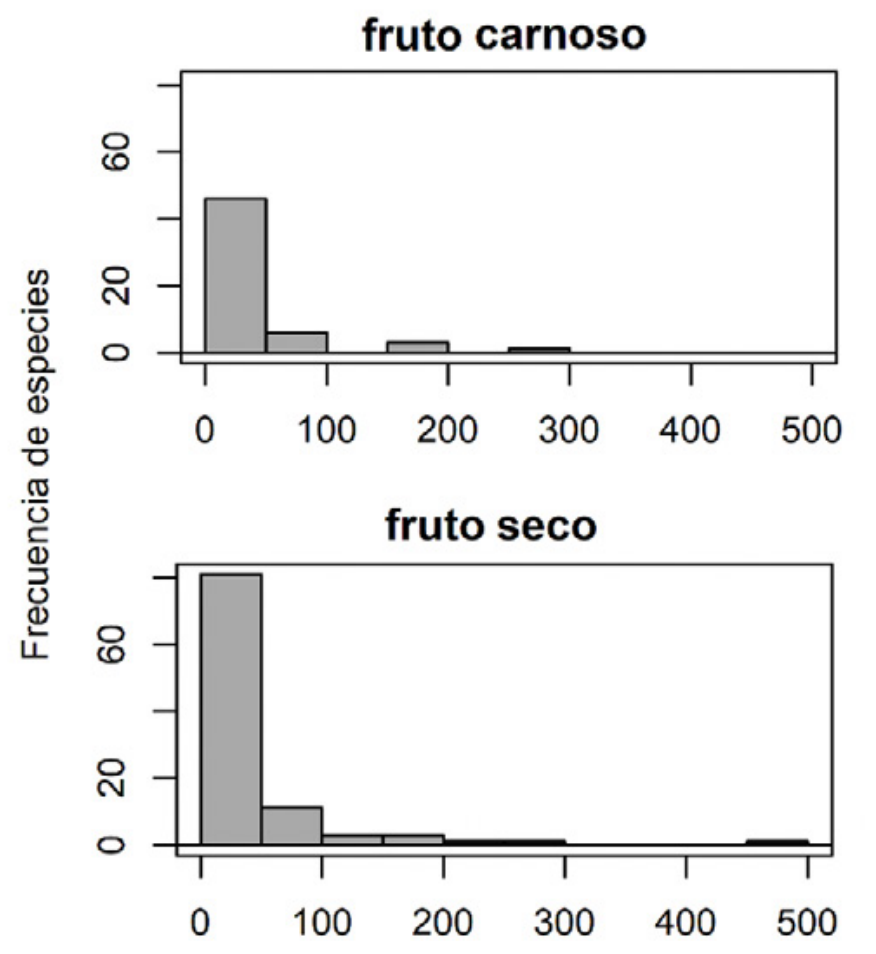

\section{Número de semillas/fruto}

Figura 5. Frecuencia de especies según el número de semillas por fruto en base al tipo de fruto.

\section{CONCLUSIONES}

Existe un amplio rango de variación en el número de semillas por fruto entre las especies leñosas distribuidas en Ecuador. Sin embargo, la tendencia del $57 \%$ de las especies es producir menos de 10 semillas por fruto, 10 que implicaría un mayor esfuerzo para la recolección de semillas con fines de conservación ex situ, especialmente en los bosques secos donde el $61 \%$ de las especies contiene en sus frutos entre 1 a 10 semillas.

A pesar de no existir diferencias significativas del número de semillas por fruto entre el tipo de fruto, hábito y entre todos los hábitats de las especies (a excepción del bosque húmedo y bosque seco), es importan- te considerar los resultados para optimizar jornadas de recolección de semillas de especies forestales. Además, los resultados podrían ayudar a complementar discusiones en futuros estudios sobre ecología de semillas que involucren rasgos morfológicos en especies forestales.

\section{REFERENCIAS BIBLIOGRÁFICAS}

Ayala-Cordero, G., Terrazas, T., López-Mata, L., y Trejo C. (2004). Variación en el tamaño y peso de la semilla y su relación con la germinación en una población de Stenocereus beneckei. Interciencia, 29(12), 692-697.

Baker, K., Richards, A. J., y Tremayne, M. A. (1994). Fitness constraints on ower number, seed number and seed size in the heteromorphic species Primula farinosa L. and Armeria maritima (Mill.) Willd. New Phytologist, 128, 562-570.

Brancalion, P. H. S., y Rodrigues, R. R. (2014). Seed size-number trade-off in Euterpe edulis in plant communities of the Atlantic Forest. Scientia Agricola, 71(3), 226-231. doi: 10.1590/S010390162014000300007

Dalling, J. W. (2002). Ecología de semillas. In M. Guariguata y G. Kattan (Eds.), Ecología y conservación de bosques neotropicales (pp. 346-375). Costa Rica: Ediciones LUR.

Gentry, A.H. (1982). Patterns of Neotropical plants species diversity. Evolutionary Biology 15,1-84.

Gritti, E. S., Cassignat, C., Flores, 0., Bonnefille, R., Cha- lié, F., Guiot, J., y JoIly, D. (2010). Simulated effects of a seasonal precipitation change on thevegetation in tropical Africa. Climate of the Past, 6, 169-178.

Hernández, V., López, E., R. G., Sánchez, P., Villarreal, R., M., Parra, T., S., Porras, F., y Corrales Madrid, J. L. (2008). Variación fenotípica entre y dentro de poblaciones silvestres de chile del noroeste de México. Revista Fitotecnia Mexicana, 31(4),323-330

Jakobsson, A., y Eriksson, 0. (2000). A comparative study of seed number, seed size, seedling size and recruitment in grassland plants. Oikos. 88(3), 494-502.

Kshitij, M. (2012). Variation in Fruit Morphological Traits of Jatropha curcas at Stand and Tree Level. Indian Forester, 12(3), 173-178.

Lavorel, S., y Garnier, E. (2002). Predicting changes in community composition and ecosystem functioning from plant traits: revisiting the Holy Grail. Functional Ecology, 16, 545-556. doi: 10.1046/j.1365-2435.2002.00664.x

Lohbeck, M., Lebrija, E., Martínez, M., Meave, J., Poorter, L., y Bongers, F. (2015). Functional Trait Strategies of Trees in Dry and Wet Tropical Forests Are Similar but Differ in Their Consequences for Succession. PLOS ONE, 10(1), e0123741. doi.org/10.1371/ journal.pone.0123741

López, C., Peña, C. B., Reyes, J. A., Aguirre, J., Ramírez, Soto, R., y Jiménez, J., (2015). Inter- and intra-specific variation in fruit biomass, number of seeds, and physical characteristics of seeds in Opuntia spp., Cactaceae. Genetic Resources and Crop Evolution, 62, 1205-1223. doi.org/10.1007/s10722-015-0223-9

Makkar, P., Martinez, J., y Becker, K (2008). Variations in Seed Number per Fruit, Seed Physical Parameters and Contents of Oil, Protein and Phorbol Ester in Toxic and Non-Toxic Genotypes of Jatropha curcas. Journal of Plant Sciences, 3: 260-265.

Montejo, L., Sánchez, J., Muñoz, B., y Gamboa, A. (2015). Caracterización de semillas de un bosque siempreverde tropical del oeste de Cuba: Correlaciones ecológicas entre rasgos. Bosque, 36(2), 211-222.

Peña, J. F., Ayala, J. D., Fischer, G., Chávez, B., Cárdenas-Hernández, J. F., y Almanza, P. J. (2010). Relaciones semilla-fruto en tres ecotipos de uchuva (Physalis peruviana L.). Agronomía Colombiana, 4(1), 43-54. 
Ramirez, N. (1993). Producción y Costo de Frutos y Semillas Entre Formas de Vida. Biotropica, 25(1), 46-60. doi: 10.2307/2388978

Romero, J., y Pérez, C., (2016a). Rasgos morfológicos regenerativos en una comunidad de especies leñosas en un bosque seco tropical tumbesino. Revista de Biología Tropical, 64(2), 859-873. doi: 10.15517/rbt.v64i2.20090

Romero, J., y Pérez, C. (2016b). Rasgos morfológicos de semillas y su implicación en la conservación ex situ de especies leñosas en los bosques secos tumbesinos. Ecosistemas, 25(2), 59-65. https://doi.org/10.7818/ECOS.2016.25-2.07

Romero, J., (2018). Seed conservation: An alternative to store germplasm and recover threatened Ecuadorian forests. Neotropical Biology and Conservation, 13(1), 74-85. doi.org/10.4013/ nbc.2018.131.09

Romero, J., y Cueva-Ojeda, D N. (2020). Tamaño de semillas y germinación de Pernettya prostrata (Ericaceae): una especie del páramo andino. Caldasia, 42(2), 326-329. https://dx.doi.org/10.15446/ caldasia.v42n2.77247.

Sharma, S., y Kumar, A. (2013). Variability studies of fruit and seed characteristics in Jatropha (Jatropha curcas L) in Himachal Pradesh. International Journal of Farm Science, 3(1), 70-76.

Vaughton, G., y Ramsey, M. (1998). Sources and consequences of seed mass variation in Banksia marginata (Proteaceae). Journal of Ecology, 86(4), 563-573. doi: 10.1046/j.13652745.1998.00279.x

Venable, D. L., y Rees, M. (2009). The scaling of seed size. Journal of Ecology, 97(1), 27-31. doi: 10.1111/j.1365-2745.2008.01461.x.

Wolf, L., Hainsworth, F.R., Mercier, T., y Benjamin R. (1986). Seed size variation and pollinator uncertainty in Ipomopsis aggregata (Polemoniaceae). Journal of Ecology, 74,361-371. 